\title{
Study on the Legalization of Minor Internet Security
}

\author{
Pengfei Ji \\ Department of Foundation, Northeast Petroleum University at Qinhuangdao, Qinhuangdao, China \\ Email: guicaitang@163.com
}

Received 26 January 2015; accepted 4 March 2015; published 10 March 2015

Copyright (C) 2015 by author and Scientific Research Publishing Inc.

This work is licensed under the Creative Commons Attribution International License (CC BY). http://creativecommons.org/licenses/by/4.0/

c. (i) Open Access

\begin{abstract}
As a virtual place with the feature of double-edged sword, the adverse effects the Internet has on the minors can be seen not only from the information it carries but also from a series of Internet products developed based on the virtual Internet environment. Since these adverse effects have imperceptibly endangered the minors who are in their critical growth period from all aspects, we must learn from the foreign advanced legislative experience and take measures including the definition of legislative regulation, improvement of guardianship system, establishment of regulatory authorities and so on to protect the legitimate rights and interests of the minors.
\end{abstract}

\section{Keywords}

Internet Security, Minors, Protection Mechanism, Supervision

\section{Introduction}

With the rapid development of modern science and technology, the Internet is advancing rapidly, and keeps extending into and affecting people's life at all levels. The Internet has become an indispensable virtual place for people to have a knowledge of the current social situation and obtain information, and yet it is the virtual place that has imperceptible effects on the thought environment, growth environment and values of the minor group in the process of its development and dissemination of information, among which, some adverse effects may cause harm to the growth and development of the minors.

The relevant foreign scholars have researched the minors' Internet security education for a long time and investigated the Internet information activities the minors conducted more specifically and more profoundly. Kimberly S. Young, a professor at American University of Pittsburgh, has been working on the adolescent Internet addiction since 1996. United States Congress has passed four laws regulating the Internet security from 1996 to 2000. Niccrowe, a professor at Brunel University in UK, has carried out research on adolescent Internet 
addiction in 2007. The European Union has proposed and implemented three Internet safety strategies at a cost of 38 million euros since 1999. Recently, Walrave and Lenaerts, professors at University of Antwerp in Belgium, have suggested, through their research that $92.8 \%$ of minors aged 12 to 18 access to Internet at home, and Internet is being widely used by minors. Since most of minors are experiencing the initial stage of formation of value and deficient in ability to recognize and discriminate information, they are highly vulnerable to the potential Internet risk or easily exposed to harmful information, pornographic materials and violent information, causing concern of many parents and schools over the adverse effect of Internet information on the minors. The research by Livingstone, a professor at London School of Economics and Political Science, has indicated that the minors tend to overestimate their information skills and ability to discriminate information, and the overconfidence may reduce their vigilance, cause a distraction, and then put them at risk of being misled by pornographic, violent and other harmful information. De Moor, a Dutch scholar and professor, has concluded from his research that the minors may be exposed to some potential risks in the process of using the Internet. These risks can be roughly summarized as follows: content risk, contact risk and business risk, and all these potential risks will have directly or indirectly adverse effect on the growth of the minors.

In China, the China Internet Network Information Center began to publish "Statistical Report on Internet Development in China” twice a year since 1997. The Center for Social Development, Chinese Academy of Social Sciences is also engaged in the research on the topic of "China Internet Project". At the same time, many domestic scholars have also published their relevant papers and topics to deepen the investigation and research on the minors Internet security (Han, 2010).

\section{The Adverse Effects of Internet on the Minors}

\subsection{The Emergence of Harmful Information in Large Amount Has Caused Serious Material and Mental Damage to the Minors}

Many websites carrying vulgar contents have emerged with the gradual advancement and improvement on the digital development of the Internet, in consequence, a great deal of unhealthy images, videos, text messages and so on pop through hyperlinks, which catches the Internet users off guard, and directly causes the frequent harassment against the minors from vulgar websites. The minor may fail to resist it once frequently exposed to such vulgar information, and be induced to commit a crime in the pursuit of excitement.

\subsection{The Widespread Online Game Culture Affects the Healthy Development of the Minors in Personality and Mentality}

Good cultural products have an undoubted role in establishing the right value of the minors. Most of the online games generated from the Internet are produced in Japan, South Korea, Europe and US, and the minors may unknowingly adapt themselves to the "negative" foreign culture when playing these popular games, and yet the above "negative" culture will significantly hinder the formation of right view of world and view of life for the minors who are in the stage of unstable growth in all respects.

\subsection{The Personal and Property Safety of the Minors Are under Threat If They Are Addicted to Cybercafe and Other Business Places}

The common problems existing in cybercafe business place include personnel in all kinds, poor environmental condition, inadequate supporting facility, incomplete filtering software and delayed update. Since the cybercafe is generally a leisure place for idlers, it is conceivable how serious the harm will be to the minors when exposed to the cybercafé.

\subsection{The Minors Internet Addiction Has Serious Effect on Their Health and Future Development}

The minors Internet addiction has become a serious social problem, which may cause the minors to suffer from sleep deprivation, aprosexia, impaired vision and other symptoms, what's worse, severely affect the healthy development of body function, learning, family and social life, make it difficult to achieve the socialization of the minors and impede their future development of high quality (Li, 2013), and these disadvantages will indirectly 
affect the original social, family stability and order, and break its original ideal state.

\section{Inadequate Legalization of Internet Security in China}

First, the special provisions for the minors Internet security protection are too less and principled. The Article 33 in the "Law on the Protection of Minors" is the only provision to specially regulate the Internet security, stating that the State shall take measures to prevent the minors Internet addiction and encourage the development of new technology to prevent the minors Internet addiction. As the only one article where the Internet security is separately specified in "Law on the Protection of Minors", this article just provides the principle regulations in the absence of substantive content, not only adding difficulties in the operation but wasting the statute resources. For another example, the relevant provisions forbidding the minors from entering the cybercafe and other business places, although developed as a formal decree, are lack of appropriate channels and measures for complaint, denouncement, supervision and control, making it difficult in practice to effectively implement these provisions.

Second, the regulations for Internet supervision at the legislative level are quite weak. As we can see from the existing legislation, the related mechanism of Internet supervision has not been formed yet. Significant flaws exist at legislative level in the virtuous detection of Internet information, provisions for online games admittance approval and grading, supervision over cybercafe business and so on.

\section{Learn from the Foreign Legislative Experience in Minors Internet Security}

\subsection{Rules and Regulation of "Harmful Information"}

Japan has enforced the "Law of Countermeasures on Bad Websites" since April of 2009 whereby "harmful information" is explicitly defined as any content obstructive to the healthy growth of the minors, including sex, violence, bullying, drug, or content encouraging the minor delinquency, in addition, under this law, any Internet operator must delete "harmful information" from the Internet immediately or confirm visitor's age using password login authentication membership system or other ways once the harmful information is discovered.

The "Communications Decency Act" and "Child Online Protection Act" of the United States have strict rules on the "harmful information" on Internet environment endangering the physical and mental health of the minors and impose severe punishment on the law violators. "Communications Decency Act" imposes a penalty up to $\$ 25,000$ or imprisonment up to two years or cumulative punishment on anyone knowingly uses an interactive computer service and electronic device to produce, abet, spread materials that are obscene or indecent (including comment, request, suggestion, proposal, image or others) in a manner available to a person under the age of 18. "Child Online Protection Act" requires all commercial pornographic websites to restrict their sites from access by minors under the age of 17 to material harmful to minors which is defined as material showing nudity and sexual act image and words in lack of literary, artistic, political and scientific value with seriousness.

The "Child Internet Safety Strategy" is a law in the United Kingdom formulated and enforced in March of 2008, requiring all Internet service providers not only to actively shield information harmful to minors but set up safety tips prominently on sites; at the same time, all websites shall properly deal with the sensitive topics about minors and provide related helpful web pages and software available through link to ensure the minors are kept away from disturbance and harm from harmful information.

The "Youth Protection Law" of the Republic of Korea is a law introduced with a series of relevant provisions to show the importance the country has attached on and the protection for security rights of minors exposed to Internet information. The law clearly states all portals and news sites shall not contain any material pornographic or unsuitable for minors, and requires all cybercafes, libraries, schools and other public places to install filtering software to ensure that the minors can obtain healthy information.

\subsection{Regulation on Classification of "Online Game"}

At present, Japan, South Korea and other Asian countries have developed a relatively complete online game grading system whereby online game products are classified by grading to fit different groups so as to achieve the purpose of restraining certain game products from flowing to the group of minors.

The online game grading system in Japan greatly depends on what age the pornographic content of the game product itself is suitable for. The grading method can be described as a method combining "distinction by age” 
with “distinction by game content”. The method of “distinction by age” is provided with four levels including suitable for all ages, over the age of 12, over the age of 15 and over the age of 18, and the results of examination and grading are indicated on the packaging boxes of games with a grading label to guide game players when buying games. The method of "distinction by game content" is a method to indicate the characteristics of game contents, such as adult content, sex, violence, terror, smoking, drinking, gambling, crime, drugs, dirty talk and others so as to guide parents and game players to when buying online game products.

The game grading system in South Korea is featured with user grade mark and warning text. The user grade mark, same as that in Japan, is divided into four grades: suitable for all ages, over the age of 12, over the age of 15 and over the age of 18, and added with different backgrounds to highlight its importance; the warning text is the text for the protection of minor, saying that the game is designed for users over the age of 18 , and no minor is allowed to use it.

\subsection{Restriction against "Cybercafe Management"}

The restrictions on "cybercafe management" mainly refer to the relevant provisions to restrict and regulate the operating service activities the offline service providers conduct (cybercafe operators). The "Child Protection Act in Public Places” of Germany prohibits any cybercafe operator to provide the minors with any game software that may cause harm to their physical and mental health and imposes criminal sanction up to 15 years of imprisonment on any person responsible for the transmission of pornographic materials through cybercafe. South Korea requires all “general Internet” (Valcke, 2011), especially cybercafe business places to interrupt violent and sexual content automatically, that is, cybercafe and other relevant business places shall install powerful interrupt software in strict accordance with requirements to prevent the Internet user group under 18 from accessing to such harmful information.

\section{Improve the Protection Mechanism of Minor Internet Security in China}

\subsection{Define the Legislative Regulations on Minor Internet Security in China}

1) The "harmful information" on the Internet shall be defined specifically, and punishment shall be imposed on the subject of liability who conducted illegal activities with reference to laws and regulations in Japan and the United States (Xue, 2010).

2) The provisions on minor Internet security protection specified in a variety of laws and regulations shall be integrated together, and the online games, audio-visual programs and other Internet culture products shall be strictly defined on whether it is healthy and what age it is suitable for. At present, the "Law on the Protection of Minors", the "Interim Measures for the Administration of Online Games" and the "Internet Audio-visual Program Service Regulations” are the three main laws to regulate the online games and audio-visual programs and other Internet culture products, so the online games and audio-visual programs and other Internet cultural products shall be provided with grading and standardizing system as soon as possible and classified in strict accordance with their respective grading and standardizing systems which can be established with reference to the online games grading and standardizing system in Japan and South Korea (Wang et al., 2013).

3) The Article 33 in the "Law on the Protection of Minors" should be modified to further explain that the State should increase the budget on Internet technology development, provide technical support for the R \& D personnel and confer the patent right on the person who developed the new technology to prevent minor Internet addiction so as to achieve the ultimate purpose of protection for the right of minor Internet security (Wang \& Yu, 2007).

4) Strengthen the legislation on Internet supervision. Internet supervision includes not only the supervision over communications providers or Internet service providers but the supervision over cybercafe business places.

\subsection{Improve the Guardianship System of Minor Internet Activities}

The monitoring and care of family members are a key factor for the minors to access Internet safely and rationally. Parents should play a role in guiding the minors to grow up healthily in the online world by taking advantage of the guide and control they have. They are expected to have knowledge of computer and Internet, communicate actively with their children and tell them to be careful not to reveal their personal information into the 
Internet and inform their parents timely if there is any problem. Schools shall shoulder the important responsibility for Internet supervision to correctly guide and educate the minors. As a social organization responsible for systematical impartment of knowledge and skills, schools should effectively function as the second classroom (Zhang \& Huang, 2014).

\subsection{Establish Regulatory Authorities of Minor Internet Security}

1) National regulatory authorities should supervise over the implementation of family guardianship and school supervision

For the supervision over the family guardianship, regulatory authorities should pay regular visit to the families of minors, ask for information by means of investigation and take advice in order to supervise whether the parents have fulfilled their obligation of Internet security supervision over their children. For the supervision over schools (Xu, 2011), regulatory authorities should conduct overall and regular inspection of safe use of campus computer, actively release the latest green online software to schools and supervise the installation of software to the schools. The regulatory authorities shall take it seriously if the minors get access to harmful information which has been penetrated into campus as a result of software system bugs.

2) Regulatory authorities should strengthen the supervision over the dissemination of Internet information in order to clean up the Internet environment at the source

a) Encourage and promote Internet technology industry and employees to develop green software good for Internet access by minors and release to users the economical, easy-to-installation and effective monitoring tools so as to intercept the invasion of harmful information.

b) Add the detected bad websites to the blacklist and cooperate with technical personnel for the development of green software used to prevent the minors from accessing to and browse the websites in blacklist.

c) Enhance the examination and verification of game software grading, and the game software unsuitable for the minors shall be clearly marked.

d) With the support of Internet technology, regulatory authorities should set up a special supervision website with a given status and responsibilities for the purpose of allowing the majority of Internet users to be involved in the supervision for the purpose of the more extensive supervision over minor Internet security.

\section{Conclusion}

In summary, since the minors Internet problem is a complex social problem, it is difficult to achieve good results only relying on government management, and it is preferable to mobilize all sectors of society, and schools, parents and communities are expected to work together for joint participation and supervision. First of all, government should associate the minor Internet management with social governance. Families and schools are not only linked closely with the minors' life, but also responsible for routine care and management. They are then supposed to play a directly administrative role in Internet management. However, many schools and parents neglect the minors Internet management and fail to conduct the required supervision and protection in reality, so the management responsibilities of schools, families and society should be strengthened by law for the effective fulfillment of protective obligation. Secondly, parents, schools and society, working as the main force of social supervision, are supposed not only to support and cooperate with the government administration but also to play a role of supervision using legal tools, to monitor the government administration according to the law and to make complaints or suggestions on where the government is performing poorly.

\section{References}

Han, J. F. (2010). Internet Security Protection for Minors-With Reference to the Combination of Internet Legal System with Family Supervision in Japan. Academic, 6.

Li, Y. (2013). Talking about the Era of Network Security and Protection of Personal Information. Electronic World, 8.

Valcke, M., Wever, B. D., Van Keer, H. et al. (2011). Long-Term Study of Safe Internet Use of Young Children. Computers \& Education, 57, 1292-1305. http://dx.doi.org/10.1016/j.compedu.2011.01.010

Wang, Y., Hong, W. D., \& Wang, Z. (2013). Research on the Network Information Behavior of Foreign Minors and Its Enlightenment. The Construction of the Library, 9.

Wang, Z. Q., \& Yu, J. M. (2007). Network Security in the Protection of Minors Study. Information Network Security, 10. 
Xu, Z. H. (2011). Discussion and Analysis on Child Internet Safety Strategy of United Kingdom. Academy, 2.

Xue, W. (2010). Study on Issues of Internet Security Protection for Minors. Journal of Beijing Youth Politics College, 3.

Zhang, L., \& Huang, W. L. (2014). Discussion on the Legal Protection of Rights of Minor Internet Security. Journal of Fujian Normal University, 5. 